\title{
Agradecimentos por financiamento na produção científica brasileira representada na Web of Science
}

\author{
Gonzalo Rubén Alvarez \\ Mestre; Universidade Federal do Rio Grande do Sul, Porto Alegre, RS, Brasil; \\ gonzalorubenalvarez@gmail.com \\ Sônia Elisa Caregnato \\ Doutora; Universidade Federal do Rio Grande do Sul, Porto Alegre, RS, Brasil; \\ sonia.caregnato@ufrgs.br
}

\begin{abstract}
Resumo: Agradecimentos presentes em artigos representam um fenômeno social e oferecem caminhos alternativos para o estudo da pesquisa financiada (agradecimentos de natureza "financeira") e da colaboração 'oculta' (agradecimentos de natureza "não financeira") na produção científica. Analisouse os agradecimentos por financiamento - funding acknowledgements (FA) identificados nas publicações brasileiras de 2009-2016 indexadas na Web of Science (WoS), observando características da pesquisa financiada por área. O corpus é composto por 268.779 artigos, dos quais 71,4\% incluem informações sobre agradecimentos por financiamento. A maior presença de FA em periódicos Q1, quando comparada à da totalidade de periódicos (todos os quartis) e em artigos minimamente citados, quando comparada à da totalidade de artigos sem FA minimamente citados, pode ser considerada um indicador de qualidade da pesquisa financiada no Brasil. De modo geral, a presença de FA em disciplinas de natureza teórica e social de áreas como Ciências Sociais e Humanidades é significativamente baixa em comparação às disciplinas de áreas de natureza experimental e aplicada como Física e Química e indica uma menor dependência de financiamento das primeiras. Os resultados revelam uma maior presença de FA em artigos de múltipla autoria, provavelmente como consequência da complexidade e custo financeiro das investigações no interior dos teamworks. Conclui-se que a presença de agradecimentos por financiamento nos artigos brasileiros varia conforme a área em virtude das particularidades de cada uma delas (natureza e orientação da pesquisa, perfil de publicação, estrutura organizacional etc.).
\end{abstract}

Palavras-chave: Produção científica. Agradecimentos por financiamento. Pesquisa financiada. Ciência brasileira. Bibliometria.

\section{Introdução}

No campo da bibliometria, diversas investigações têm documentado o crescimento da taxa de coautoria na ciência brasileira desde o final do século 
XX (LETA; CHAIMOVICH, 2002; VANZ; STUMPF, 2012), retratando o valor social da colaboração na pesquisa nacional. A coautoria tem sido convencionalmente aceita em estudos bibliométricos para estimar a colaboração científica em diferentes níveis: indivíduos, instituições e países. No entanto, esse indicador deve ser analisado com certa cautela, dado que, em determinadas situações e formas de interação profissional, a colaboração é indetectável por meio dessa abordagem.

$\mathrm{Na}$ avaliação científica, a coautoria é um indicador imperfeito e parcial da colaboração de pesquisa entre atores sociais que, tal como descrito por Katz e Martin (1997), se constitui em um processo complexo, de múltiplas dimensões. Nessa perspectiva, claras evidências de que uma proporção significativa de artigos peer review publicados em prestigiosas revistas internacionais de diversas áreas contém declarações formais de agradecimentos (PAUL-HUS; DESROCHERS; COSTAS, 2016) fizeram emergir novas oportunidades para a investigação da prática colaborativa desde uma visão mais ampla. Os agradecimentos, como indicadores de "subautoria" (HEFFNER, 1981) podem ser utilizados para analisar relações sociais complementares no campo científico, descobrir padrões comportamentais entre áreas e avaliar aspectos particulares da colaboração "invisível”.

Os agradecimentos fornecem indícios das influências materiais (financeiras e outras) na pesquisa, podendo ser utilizados para examinar práticas de colaboração baseadas nas relações sociais de caráter estratégico e mercadológico que permeiam a atividade científica (LATOUR; WOOLGAR, 1997). Na óptica da Sociologia da Ciência, análises quantitativas sobre padrões de agradecimentos têm sido desenvolvidas desde a década de 1970 (CHUBIN, 1975) para avaliar a dinâmica da colaboração dentro e entre grupos de diferentes disciplinas.

Por alguns anos, no contexto dos estudos bibliométricos, os agradecimentos foram relativamente negligenciados em virtude da falta de um índice similar ao Science Citation Index (SCI) da Web of Science (WoS) para coleta e estruturação das informações. Desde 2008, entretanto, observa-se um importante incremento do número de investigações, provavelmente, como 
consequência da sistemática inclusão de dados sobre agradecimentos por financiamento presentes em revistas indexadas pela WoS (PAUL-HUS et al., 2015).

Agradecimentos podem ser explorados com variados propósitos que vão desde a investigação das conexões e dependências sociocognitivas dentro e entre comunidades de discurso acadêmico (agradecimentos de natureza "não financeira") até a avaliação da pesquisa científica e a formulação de estratégias e políticas de financiamento (agradecimentos de natureza "financeira"). Em virtude de que diversos organismos de fomento são reconhecidos pelas suas contribuições durante o andamento do trabalho de pesquisa, a análise das informações que textos de agradecimentos por financiamento fornecem também pode ser útil para examinar a relação entre os subsídios dispensados e a produção de conhecimento registrado, assim como para avaliar a influência das agências financiadoras distinguidas como "colaboradoras" sobre cada trabalho publicado (RIGBY, 2011, 2013). Desde então, vários pesquisadores utilizaram dados de agradecimento por apoio financeiro processados pela WoS para analisar essas tendências e identificar os principais organismos de fomento à pesquisa em artigos científicos por especialidade médica (LEWISON; MARKUSOVA, 2010; LEWISON; ROE, 2012) e por país (WANG; SHAPIRA, 2011; WANG et al., 2012; LIU et al., 2015).

Estudos sobre produção científica que têm analisado o impacto da investigação pelo nível de apoio financeiro a projetos avaliam criticamente a importância e a utilização do rendimento de pesquisa per capita como indicador de qualidade (GILLETT, 1991) e do financiamento externo como indicador de desempenho (LAUDEL, 2005). Entretanto, um grupo mais entusiasmado de cientistas sustenta a ideia de que a quantidade de financiamento e a identidade das fontes de fomento são fatores importantes que influenciam o aumento das citações e do impacto dos artigos científicos, visto que os órgãos financiadores, geralmente, aplicam algum tipo de revisão por pares sobre os pedidos de subvenção (RIGBY, 2011).

Em particular, alguns especialistas, ao analisarem todas as publicações de 2009 abrangidas pela WoS, observaram que, de fato, os trabalhos de pesquisa 
com agradecimentos por apoio financeiro apresentam um impacto maior em relação ao resto dos artigos (COSTAS; VAN LEEUWEN, 2012). Na área da Biblioteconomia e Ciência da Informação, constatou-se o mesmo fenômeno ao examinar o impacto da produção científica financiada por subvenções e não financiada que foi publicada em 1998 em sete dos periódicos mais importantes da área (ZHAO, 2010). Publicações financiadas por patrocinadores de pesquisa prestigiosos geram um maior interesse de citação (WANG; SHAPIRA, 2011).

Outros pesquisadores analisaram em profundidade a relação entre o número de fontes de financiamento e as citações recebidas por artigos publicados em revistas científicas na área de Gastroenterologia, chegando à conclusão de que as duas variáveis estão positivamente correlacionadas, ou seja, quanto maior for o número de agências financiadoras por documento, maior será o impacto das publicações (LEWISON; GRANT; JANSEN, 2001). Entretanto, Rigby $(2011,2013)$ afirma que essa relação é mais complexa do que se supõe, podendo existir variações conforme o campo científico analisado e o tipo de organismo de financiamento envolvido nos diferentes tipos de pesquisa.

Em análise da frequência de agradecimentos por financiamento identificados nas publicações (artigos originais e revisões) espanholas de 2010 indexadas pela WoS, identificaram-se altos percentuais de publicações financiadas em campos experimentais como Química e Física e baixos percentuais de publicações financiadas em campos teóricos como Humanidades e Ciências Sociais (DÍAZ-FAES; BORDONS, 2014).

No Brasil, e em outros países em desenvolvimento, entretanto, os agradecimentos como indicadores de pesquisa financiada e colaboração é ainda um assunto pouco compreendido, merecendo ser analisado com maior profundidade e detalhamento, principalmente, através da combinação de técnicas de pesquisa que possibilitem a validação e a reconstrução de novos conhecimentos acerca das implicações sociais da prática e do comportamento que estimula o uso deles na ciência.

O estudo da presença dos agradecimentos por financiamento nos artigos brasileiros pode contribuir substancialmente para o entendimento das características da pesquisa financiada por disciplina/área no âmbito nacional. 
Nesse sentido, e apoiado na literatura, este artigo é uma continuação do trabalho desenvolvido e apresentado por Alvarez e Caregnato (2018) no $6^{\circ}$ Encontro Brasileiro de Bibliometria e Cientometria ( $\left.6^{\circ} \mathrm{EBBC}\right)$, analisando por meio de indicadores bibliométricos, os agradecimentos por apoio financeiro presentes nos artigos brasileiros do período de 2009-2016 indexados na WoS.

\section{Metodologia}

A pesquisa desenvolvida caracteriza-se como um estudo bibliométrico de nível macro de agregação e analisa a presença dos textos de agradecimento por financiamento nos artigos brasileiros indexados na base de dados multidisciplinar Web of Science (WoS) do grupo Clarivate Analytics.

A coleta de dados foi realizada em outubro de 2017, utilizando a estratégia de busca por país (CU), limitada aos índices de citação Science Citation Index Expanded (SCIE), Social Sciences Citation Index (SSCI), Arts and Humanities Citation Index (AHCI); ao tipo de documento Article e Review; ao idioma English e ao período de 2009-2016. O corpus da pesquisa é composto por artigos originais e revisões. A escolha se deve ao fato de serem normalmente os tipos de documentos que recebem maior atenção da comunidade científica, o que acaba se refletindo no número de citações que recebem em relação aos demais. O período de 2009-2016 foi selecionado pela atualidade e abrangência de informações sobre agradecimentos.

As informações oferecidas pela WoS sobre funding acknowledgements (FA) estão organizadas em três seções: funding agency (FO), que contém o nome do órgão de fomento que financiou a pesquisa; grant number (FG), que contém o número de identificação do projeto financiado; funding text (FT), que contém o texto completo dos agradecimentos. O FT inclui não apenas informações sobre apoio financeiro, mas também sobre tipos de contribuições agradecidas pelos autores de natureza "não financeira".

Como fonte de informação principal utilizou-se a seção FT, correspondendo ao texto completo sobre agradecimentos por financiamento extraído do documento original indexado pela WoS. Ressalta-se que a WoS 
processa unicamente dados referentes aos agradecimentos quando o texto de FT for registrado em inglês, indiferente ao idioma do corpo da publicação, e quando tiver alguma menção sobre financiamento recebido pelos autores do artigo.

A distribuição dos 268.779 artigos originais e de revisão brasileiros que compõem o corpus de pesquisa por disciplina baseia-se na classificação dos periódicos em categorias de assunto da WoS, ressaltando que um periódico pode ser classificado em até seis categorias diferentes. Quanto às áreas de publicação, os artigos foram organizados conforme a classificação utilizada para examinar a presença de agradecimentos com FA nas publicações espanholas de 2010 (DÍAZ-FAES; BORDONS, 2014), a produção científica do Consejo Superior de Investigaciones Científicas (CSIC) de 2011-2015 (BORDONS et al., 2016) e a completude e a precisão da WoS na extração e processamento de dados sobre financiamento (ÁLVAREZ-BORNSTEIN; MORILLO; BORDONS, 2017). Tais áreas correspondem a: Agricultura, Biologia e Meio Ambiente, Biomedicina, Ciências Sociais, Engenharia, Tecnologia, Física, Humanidades, Matemática, Medicina Clínica, Multidisciplinar e Química.

O Journal Citation Reports (JCR) 2016 da WoS foi consultado em novembro de 2017 para identificar os periódicos pertencentes ao Q1 (25\% das revistas com maior fator de impacto) por disciplina/área. Bibexcel e Microsoft Excel foram os softwares utilizados nas análises descritivas e SPSS nas análises estatísticas. O teste não paramétrico $U$ de Mann-Whitney foi usado para analisar a significância da diferença entre a média do número de autores/artigo do conjunto de publicações brasileiras com agradecimentos por financiamento (Com FA) e a média do conjunto de publicações brasileiras sem agradecimentos por financiamento (Sem FA). O teste qui-quadrado de Pearson foi utilizado para observar se existe associação entre as variáveis agradecimentos por financiamento e citação no conjunto de artigos brasileiros por área.

\section{Resultados e discussão}

A produção científica brasileira de 2009-2016 indexada na WoS é constituída por 268.779 documentos (artigos originais e revisões). A totalidade de 
publicações de pesquisadores brasileiros está distribuída em 249 categorias de assunto (disciplinas WoS), sendo que 238 contêm menções de agradecimento por financiamento (FA). $\mathrm{O}$ apoio recebido por fontes de financiamento para o desenvolvimento das pesquisas nacionais é reconhecido em uma grande quantidade de publicações que compõem o corpus desta investigação, 191.827 artigos $(71,4 \%)$ (Tabela 1). A taxa é maior do que os aproximadamente $45 \%$ encontrados para as publicações brasileiras com FA em estudos anteriores (COSTAS; LEEUWEN, 2012).

\begin{tabular}{cccc}
\multicolumn{4}{c}{ Tabela 1 - Documentos brasileiros com FA na WoS (2009-2016) } \\
\hline Ano & $\begin{array}{c}\mathbf{N}^{\circ} \text { artigos originais } \\
\text { e revisões }\end{array}$ & $\begin{array}{c}\mathbf{N}^{\circ} \text { artigos originais e } \\
\text { revisóes com FA }\end{array}$ & $\begin{array}{c}\text { \% } \\
\text { FA }\end{array}$ \\
\hline 2009 & 24.417 & 15.046 & 61,6 \\
2010 & 26.125 & 17.547 & 67,2 \\
2011 & 29.279 & 20.408 & 69,7 \\
2012 & 32.912 & 23.032 & 70,0 \\
2013 & 35.177 & 25.517 & 72,5 \\
2014 & 37.341 & 27.451 & 73,5 \\
2015 & 39.644 & 30.001 & 75,7 \\
2016 & 43.884 & 32.825 & 74,8 \\
Total & $\mathbf{2 6 8 . 7 7 9}$ & $\mathbf{1 9 1 . 8 2 7}$ & $\mathbf{7 1 , 4}$ \\
\hline \multicolumn{4}{c}{}
\end{tabular}

A expansão das publicações brasileiras e o consequente aumento do número de artigos com agradecimentos por financiamento no período de 20092016 podem ser observados na Tabela 1, comprovando-se como a ampliação de bolsas e incentivos através de projetos financiados contribuiu para o desenvolvimento da ciência no âmbito nacional. A maior proporção de agradecimentos por financiamento identificada nos artigos brasileiros de 2015 pode ter sido consequência da ampliação da cobertura de FA a partir de dito ano, quando a WoS começou sistematicamente a coletar e processar informações sobre agradecimentos por apoio financeiro dos artigos publicados em revistas abrangidas pelo SSCI, beneficiando assim, pela combinação de índices (SCIE + SSCI), o aumento do número de registros com textos de financiamento e, consequentemente, o alargamento das possibilidades de investigação sobre 
pesquisa financiada e subautoria no Brasil através de indicadores bibliométricos. A Tabela 1 também exibe um nítido aumento da proporção de publicações brasileiras com FA para o período (61,6\% em 2009 e 74,8\% em 2016). O crescimento anual do volume de FA observado tanto nas publicações mundiais (PAUL-HUS; DESROCHERS; COSTAS, 2016) quanto nacionais ratifica o rol dos órgãos de fomento à pesquisa e o valor do financiamento para a produção científica no contexto institucional.

\section{1 Áreas e disciplinas dos documentos brasileiros com ou sem FA}

A distribuição de FA por ano de publicação por área mostra que o maior número de artigos brasileiros com agradecimentos por financiamento foi publicado em revistas da área de Agricultura, Biologia e Meio Ambiente (Figura 1), revelando o alto investimento realizado pelo Brasil na pesquisa científica nesta área no decorrer dos anos, principalmente através de instituições públicas vinculadas ao Ministério da Agricultura, Pecuária e Abastecimento como a Empresa Brasileira de Pesquisa Agropecuária (EMBRAPA) (VANZ, 2009; VARGAS, 2014). Conforme o Ministério da Ciência, Tecnologia e Inovação (MCTI), cerca de 10,0\% dos dispêndios públicos do governo federal em pesquisa e desenvolvimento (P\&D) no ano de 2013 foram direcionados para investigações no âmbito da agricultura, sendo que o percentual é maior do que o destinado para a saúde (9,8\% contra 6,6\%) (BRASIL, 2013).

Outras áreas relacionadas às Ciências da Saúde (Biomedicina e Medicina Clínica), Engenharias e Ciências Exatas e da Terra (sobretudo Física e Química) também apresentaram importantes valores em termos de publicações com FA, sinalizando a eficácia dos investimentos do CNPq realizados entre 2009 e 2016 em projetos de pesquisa dessas áreas, levando em consideração a totalidade de recursos e capital efetivamente pagos no período (CNPq, 2018). 
Figura 1 - Documentos brasileiros com FA por área na WoS (2009-2016)

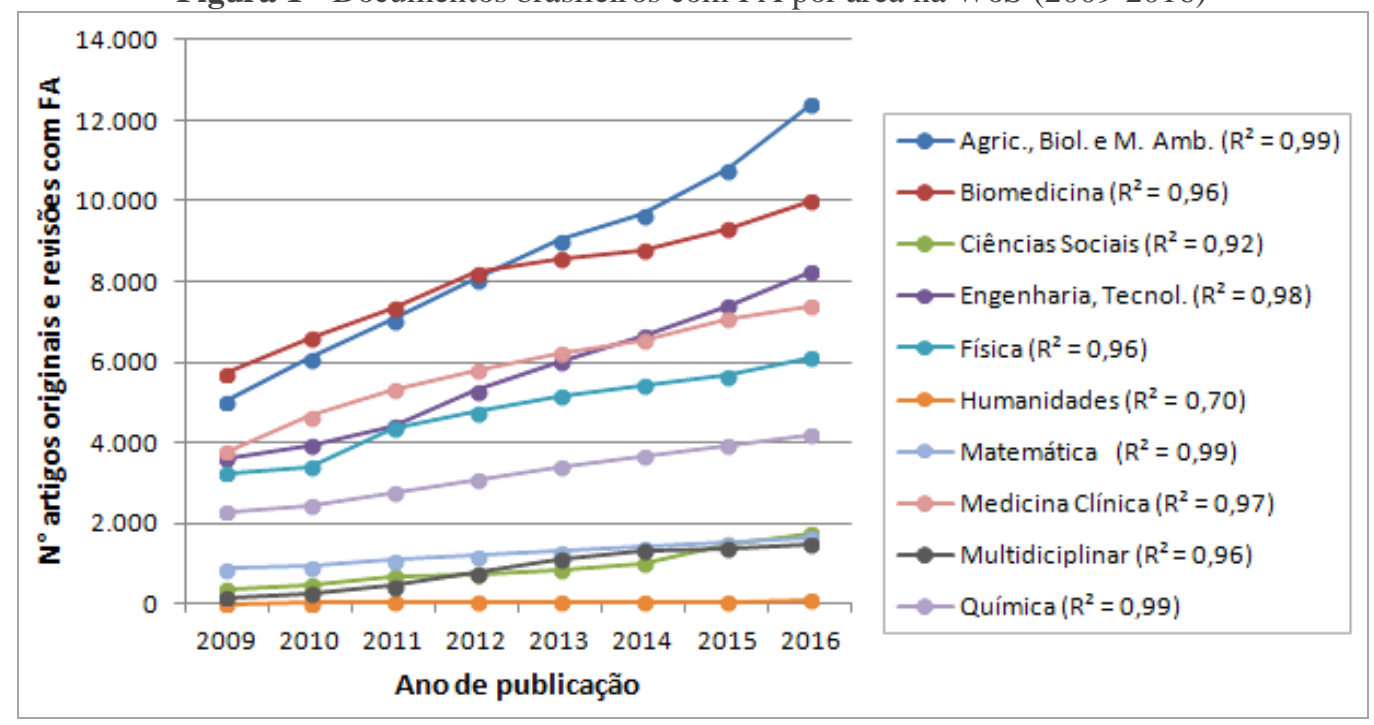

Fonte: Web of Science (2017).

Por outro lado, observam-se valores inferiores em termos de número de publicações com FA em áreas como Ciências Sociais e Humanidades, que sabidamente têm uma menor dependência de equipamentos e infraestrutura do que as "ciências teórico-conceituais" na atividade científica e, em consequência, de investimento (DÍAZ-FAES; GALINDO; BORDONS, 2013). Produções científicas derivadas de financiamento em Ciências Sociais e Humanidades acontecem de forma eventual, provavelmente em menor intensidade quando comparadas a outras áreas como, por exemplo, a Física, presumindo que o grau de autonomia de uma área depende do grau de necessidade de recursos econômicos que ela exige para se concretizar (BOURDIEU, 2004).

A presença de FA em artigos de periódico na língua inglesa publicados por pesquisadores brasileiros varia conforme as áreas do conhecimento (Tabela 2). Em áreas com Humanidades $(18,5 \%)$ e Ciências Sociais $(40,7 \%)$ a baixa presença de FA $(<41,0 \%)$ pode ser influenciada por fatores culturais vinculados à falta de tradição e obrigatoriedade para agradecer formalmente às suas fontes financiadoras (COSTAS; LEEUWEN, 2012). Valores intermediários são observados em Medicina Clínica (54,8\%). Engenharia, Tecnologia, Agricultura, Biologia e Meio Ambiente e Matemática apresentaram percentuais de FA na faixa de 70,0\% para o período analisado. A maior presença de agradecimentos por financiamento (>80\%) foi observada em quatro áreas, com destaque para as 
experimentais: Biomedicina (80,8\%), Química (85,0\%), Física $(85,4 \%)$ e Multidisciplinar (85,8\%). O alto índice de FA em campos experimentais como a Química corrobora os resultados encontrados em estudos anteriores (CRONIN; SHAW; LA BARRE, 2004; DÍAZ-FAES; BORDONS, 2014).

Tabela 2 - Documentos brasileiros com FA por área e quartil de periódico Q1 (2009-2016)

\begin{tabular}{|c|c|c|c|c|c|c|}
\hline \multirow[b]{2}{*}{ Área } & \multicolumn{3}{|c|}{ Todos os periódicos } & \multicolumn{3}{|c|}{ Periódicos Q1 } \\
\hline & $\begin{array}{l}\mathrm{N}^{\circ} \text { artigos } \\
\text { originais e } \\
\text { revisões }\end{array}$ & $\begin{array}{l}\mathrm{N}^{\circ} \text { artigos } \\
\text { originais e } \\
\text { revisões } \\
\text { com FA }\end{array}$ & $\begin{array}{l}\% \text { artigos } \\
\text { originais e } \\
\text { revisões } \\
\text { com FA }\end{array}$ & $\begin{array}{l}\mathrm{N}^{\circ} \text { artigos } \\
\text { originais } \\
\text { e revisões }\end{array}$ & $\begin{array}{l}\mathrm{N}^{\circ} \text { artigos } \\
\text { originais e } \\
\text { revisões } \\
\text { com FA }\end{array}$ & $\begin{array}{l}\% \text { artigos } \\
\text { originais } \\
\text { revisões } \\
\text { com FA }\end{array}$ \\
\hline $\begin{array}{l}\text { Agric., Biol. e Meio } \\
\text { Amb. }\end{array}$ & 88.867 & 68.102 & 76,6 & 23.867 & 20.462 & 85,7 \\
\hline Biomedicina & 79.881 & 64.576 & 80,8 & 14.162 & 12.466 & 88,0 \\
\hline Ciências Sociais & 18.066 & 7.346 & 40,7 & 5.145 & 2.886 & 56,1 \\
\hline $\begin{array}{l}\text { Engenharia, } \\
\text { Tecnologia }\end{array}$ & 60.981 & 45.562 & 74,7 & 26.139 & 20.439 & 78,2 \\
\hline Física & 44.647 & 38.146 & 85,4 & 17.382 & 15.945 & 91,7 \\
\hline Humanidades & 1.296 & 240 & 18,5 & 92 & 35 & 38,0 \\
\hline Matemática & 13.067 & 10.099 & 77,3 & 4.490 & 3.554 & 79,2 \\
\hline Medicina Clínica & 85.326 & 46.750 & 54,8 & 21.660 & 15.201 & 70,2 \\
\hline Multidisciplinar & 7.991 & 6.855 & 85,8 & 5.634 & 5.225 & 92,7 \\
\hline Química & 30.221 & 25.685 & 85,0 & 8.069 & 7.001 & 86,8 \\
\hline Total & 430.343 & 313.361 & $72,8^{1}$ & 126.640 & 103.214 & $81,5^{2}$ \\
\hline
\end{tabular}

Fonte: Web of Science (2017).

Nota: A soma total de artigos (originais e de revisão) excede o total de registros coletados visto que alguns periódicos são classificados em mais de uma área. ${ }^{1,2}$ Cálculo percentual com base na somatória do total de artigos/área. Primeiro Quartil (Q1): top 25\% da distribuição de revistas com fator de impacto indexadas pela WoS por categoria de assunto (disciplina) / área.

A taxa percentual de FA em artigos brasileiros publicados no período de 2009-2016 em periódicos Q1 (25\% das revistas com maior fator de impacto) é maior do que a do total de periódicos (todos os quartis) (81,5\% contra 72,8\%). Diferenças percentuais significativas são observadas em Ciências Sociais (56,1\% contra 40,7\%), Humanidades (38,0\% contra 18,5\%) e Medicina Clínica (70,2\% contra 54,8\%), confirmando os resultados encontrados por Díaz-Faes e Bordons (2014) em análise das publicações espanholas com FA de 2010. A maior presença (\%) de FA observada em periódicos Q1 para todas as áreas pode ser considerada um indicador de qualidade da pesquisa financiada no Brasil. 
A produção científica brasileira financiada na área Multidisciplinar é relevante não só desde a perspectiva quantitativa, mas também da qualitativa, visto que um número importante de artigos foi publicado em periódicos Q1, como Nature e Science. Tais veículos de publicação têm uma grande audiência e altas taxas de rejeição de manuscritos (GONZÁLEZ-ALBO et al., 2012), correspondendo a outra evidência da capacidade e competitividade dos cientistas brasileiros para realizarem projetos de pesquisa de ponta provenientes de financiamento interno e/ou externo em diversas temáticas de interesse internacional.

Em termos comparativos, percebe-se que áreas experimentais e mais “internacionalizadas" como, por exemplo, Biomedicina, Química e Física, são mais "privilegiadas", do que as áreas de cunho teórico como Humanidades e Ciências Sociais, não apenas no que diz respeito à cobertura pelas bases de dados, publicação em coautoria, veiculação de ideias e novidades científicas em periódicos de alto fator de impacto e acesso a infraestrutura. Elas também são "privilegiadas" no que se refere à concessão de recursos financeiros como decorrência, talvez, da sua maior participação em projetos de colaboração multinacionais envolvendo o apoio de agências de financiamento de diferentes países.

A Figura 2 apresenta a distribuição das 238 categorias de assunto, ou seja, das disciplinas da WoS das publicações brasileiras com FA por área. A presença de agradecimentos por financiamento pode variar conforme as características da disciplina (objeto de estudo, método de investigação, estrutura organizacional em rede, natureza da pesquisa: teórica/experimental) e do tipo de órgão de fomento envolvido na investigação científica. Observa-se baixa dispersão (variação percentual) de FA por disciplina WoS em áreas de natureza experimental como Física e Química (entre 70\% e 92\%), com destaque para as disciplinas: Física de Altas Energias (FAE) (91,8\%) e Química Orgânica (91,3\%). Altos índices de FA são esperados em FAE como decorrência do alto custo da pesquisa e complexidade dos experimentos no seio das grandes colaborações (teamworks) internacionais (CHOMPALOV; GENUTH; SHRUM, 2002). A baixa dispersão de FA encontrada em áreas experimentais vinculadas 
às Ciências Exatas e da Terra corrobora resultado anterior de Díaz-Faes e Bordons (2014), ao analisarem a presença de FA nos artigos espanhóis de 2010.

Figura 2 - Documentos brasileiros com FA por disciplinas WoS/área (2009-2016)

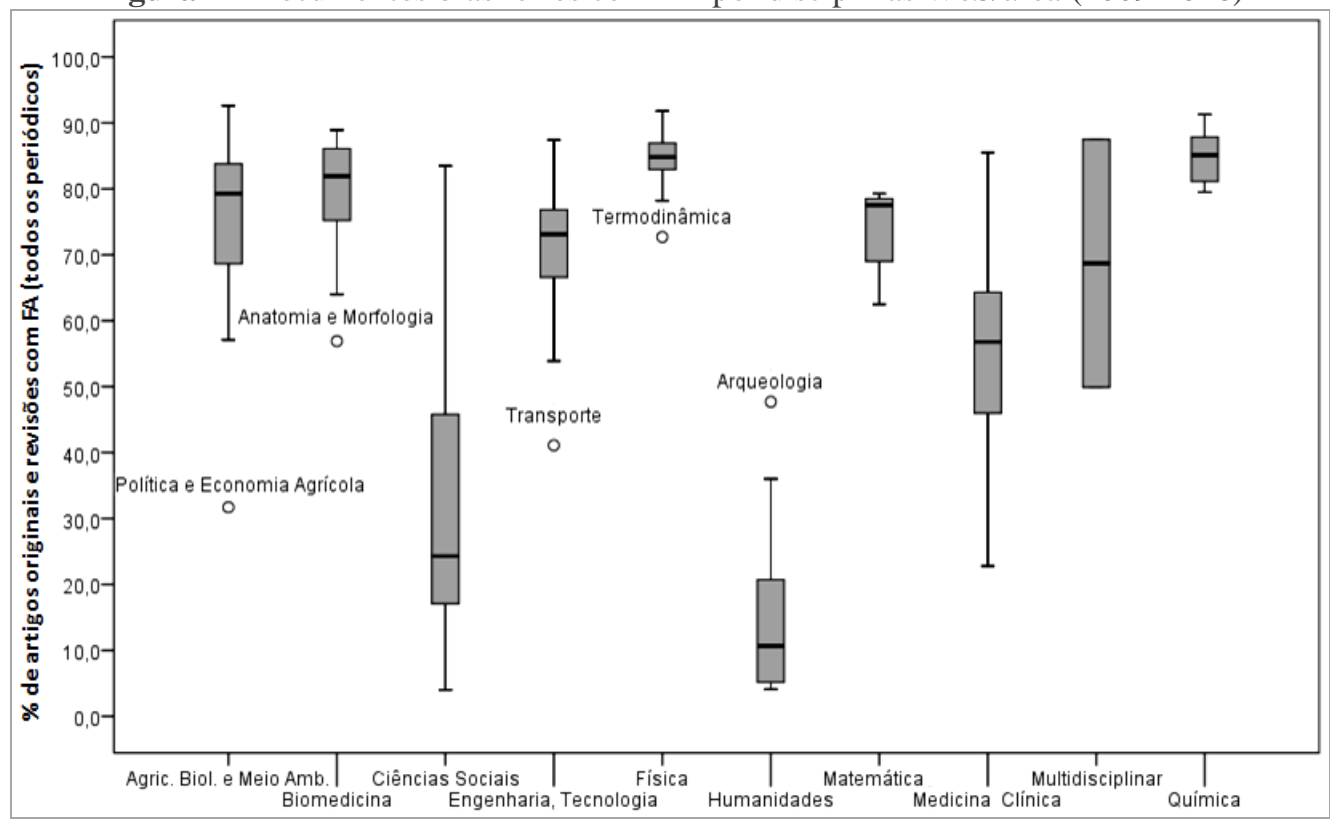

Fonte: Web of Science (2017).

Percebe-se um alto nível de dispersão de disciplinas com FA em: Agricultura, Biologia e Meio Ambiente (92,6\% em Biologia Evolutiva e 31,7\% em Política e Economia Agrícola), Biomedicina (88,9\% em Biofísica e 56,9\% em Anatomia e Morfologia), Ciências Sociais (83,5\% em Psicologia Biológica e 4\% em Psicologia e Psicanálise), Engenharia, Tecnologia (87,4\% em Nanociência e Nanotecnologia e 41,1\% em Transporte), Humanidades (47,7\% em Arqueologia e 4,1\% em Religião), Medicina Clínica (85,5\% em Toxicologia e 22,8\% em Otorrinolaringologia). A distribuição percentual mostra baixa dispersão de artigos com FA por disciplina em Matemática (62,5\% a 79,3\%). O caso da área Multidisciplinar deve ser analisado com certa cautela, uma vez que apenas inclui duas categorias com FA (DÍAZ-FAES; BORDONS, 2014).

\section{2 Áreas, disciplinas e autores dos documentos brasileiros com ou sem FA}

A relação entre a presença de FA nas publicações brasileiras de 2009-2016 e o número de autores/artigo é apresentada na Tabela 3, observando-se variações 
nos valores da média quando comparados ao conjunto de artigos Sem FA por área. Diferenças consideráveis entre a média de autores/artigos Com FA e Sem FA $(146,5$ contra 25,8) foram constatadas na área da Física, sugerindo que a presença de FA tende a aumentar com o número de pesquisadores envolvidos na investigação, principalmente na subárea da Física de Altas Energias (e disciplinas tematicamente próximas como Astronomia e Astrofísica e Física Nuclear), na qual a conformação de teamworks, a colaboração internacional e a utilização conjunta de infraestrutura e laboratórios se torna essencial devido à complexidade e custo financeiro dos experimentos com aceleradores de partículas (ALVAREZ; VANZ; BARBOSA, 2017). Verificou-se que a diferença entre as médias do número de autores/artigo dos dois conjuntos de publicações analisados (Sem FA e Com FA) é estatisticamente significativa em todas as áreas (Teste U de Mann-Whitney, p<0,05).

Tabela 3 - Média de autores nos documentos brasileiros com FA por área (2009-2016)

\begin{tabular}{llll}
\hline \multirow{2}{*}{ Área } & \multicolumn{2}{c}{$\mathbf{N}^{\circ}$ de autores/artigo (originais e revisões) } \\
\cline { 2 - 3 } & \multicolumn{2}{c}{ Média \pm Desvio Padrão } & \\
\cline { 2 - 3 } & Sem FA & Com FA & -valor \\
\hline Agric., Biol. e Meio Amb. & $5,3 \pm 4,3$ & $5,4 \pm 5,4$ & $<0,05$ \\
Biomedicina & $6,5 \pm 20,8$ & $7,3 \pm 8,0$ & $<0,05$ \\
Ciências Sociais & $3,8 \pm 3,2$ & $5,6 \pm 4,4$ & $<0,05$ \\
Engenharia, Tecnologia & $4,7 \pm 29,9$ & $7,5 \pm 80,1$ & $<0,05$ \\
Física & $25,8 \pm 207,6$ & $146,5 \pm 549,5$ & $<0,05$ \\
Humanidades & $1,9 \pm 3,3$ & $3,1 \pm 3,0$ & $<0,05$ \\
Matemática & $2,5 \pm 1,2$ & $2,7 \pm 1,5$ & $<0,05$ \\
Medicina Clínica & $6,0 \pm 7,0$ & $7,9 \pm 18,8$ & $<0,05$ \\
Multidisciplinar & $8,5 \pm 87,3$ & $9,4 \pm 62,1$ & $<0,05$ \\
Química & $5,2 \pm 2,9$ & $5,4 \pm 2,6$ & $<0,05$ \\
\hline
\end{tabular}

Fonte: Web of Science (2017).

A ideia de que a pesquisa colaborativa é comumente beneficiada pelas agências de fomento foi concebida na literatura por estudos anteriores que examinaram o impacto da produção científica financiada por subvenções e não financiada na área de Biblioteconomia e Ciência da Informação (ZHAO, 2010) e a presença de agradecimentos por financiamento nas publicações espanholas (DÍAZ-FAES; BORDONS, 2014). 
Da mesma maneira, é interessante ressaltar que o maior nível de colaboração observado na pesquisa brasileira financiada de 2009-2016 pode ser uma das principais razões para explicar a prevalência de artigos com FA em periódicos Q1 (Tabela 2) e de artigos com FA minimamente citados (Tabela 4). Dado que uma correlação positiva forte entre o número de autores/artigo e o impacto foi comprovada por diversos pesquisadores em estudos anteriores (LOW et al., 2014). Finalmente, Bozeman e Lee (2003) acrescentam que a colaboração não apenas tem influência na produção científica e na visibilidade da pesquisa, mas também na obtenção de financiamento através de bolsas, convênios bilaterais ou multilaterais, programas e políticas de investigação.

A relação entre o número de autores/artigo e a frequência de FA nas publicações brasileiras de 2009-2016 indexadas na WoS é exibida na Figura 3, identificando-se variações na linha de crescimento por área. De maneira mais clara, percebe-se que nas áreas de Ciências Sociais, Humanidades e Medicina Clínica a relação entre o percentual de artigos com FA e o número de autores/artigo se ajusta melhor a um modelo de regressão linear, coincidindo com resultados de pesquisas anteriores (DÍAZ-FAES; BORDONS, 2014). Embora não tão acentuada quanto nas três áreas anteriormente citadas, uma correlação linear também é percebida para a área de Engenharia, Tecnologia. No resto das áreas, o maior aumento em termos percentuais de FA é verificado entre a parcela de artigos com um autor e com dois autores, mostrando baixo incremento posteriormente. A área de Matemática apresenta um padrão misto, com acréscimos e decréscimos na taxa de FA conforme aumenta progressivamente o número de autores/artigo envolvidos na investigação científica. De modo geral, os indicadores da pesquisa apontam que baixos níveis de colaboração e a autoria única tendem a registrar menores percentuais de agradecimentos por financiamento, enquanto na múltipla autoria, a presença de FA é maior, confirmando os dados encontrados anteriormente por outros pesquisadores ao analisarem os textos de agradecimento nas publicações mundiais de 2009 (COSTAS; VAN LEEUWEN, 2012). 
Figura 3 - Número de autores/artigo nos documentos brasileiros com FA por área (2009-2016)

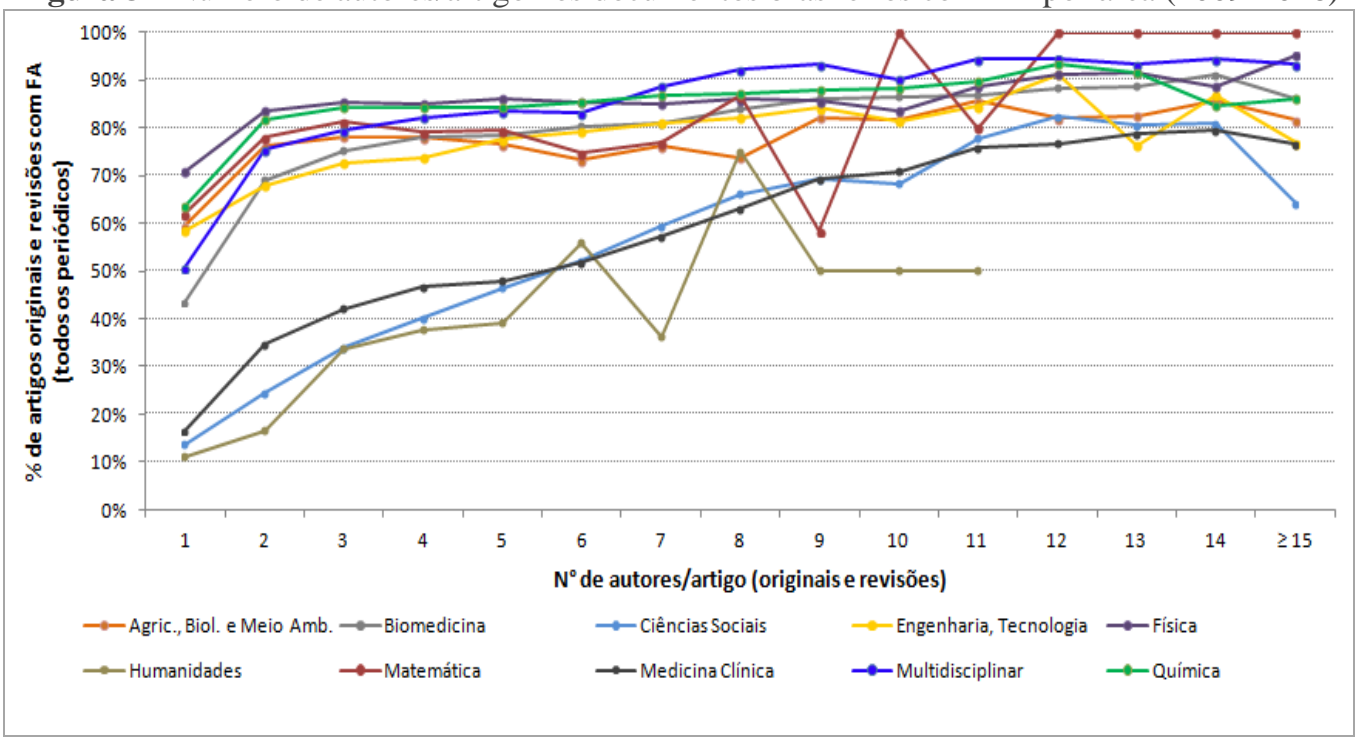

Fonte: Web of Science (2017).

\subsection{Citações nos documentos brasileiros com ou sem FA}

Verificou-se que o percentual do conjunto de artigos brasileiros Com FA citados é superior ao do conjunto de artigos brasileiros Sem FA citados em todas as áreas (Tabela 4). Após análise prévia das frequências observadas, notou-se que existe associação entre as variáveis agradecimento por financiamento e citação ou, equivalentemente, que há diferença estatisticamente significativa entre a proporção de artigos Com $F A$ citados e a proporção de artigos Sem $F A$ citados para todas as áreas (Teste $\mathrm{x}^{2}$ qui-quadrado de Pearson, $\left.\mathrm{p}<0,05\right)$. A quantidade de financiamento e a reputação das fontes de fomento parecem ser fatores relevantes que influenciam no aumento do número das citações e do impacto dos artigos, visto que os órgãos financiadores, geralmente, aplicam algum tipo de revisão por pares sobre os pedidos de subvenção (RIGBY, 2011).

Tabela 4 - Citações vinculadas aos documentos brasileiros com FA por área (2009-2016)

\begin{tabular}{cccc}
\hline Agric., Biol. e M. Amb. & \multicolumn{2}{c}{ Agrad. financiamento } & \\
\cline { 2 - 3 } Citação & Com FA & Sem FA & Total \\
\hline Artigos citados (\%) & $56.859(83,50)$ & $15.209(73,24)$ & $72.068(81,10)$ \\
Artigos não citados (\%) & $11.243(16,50)$ & $5.556(26,76)$ & $16.799(18,90)$ \\
\hline Total & 68.102 & 20.765 & 88.867 \\
\hline Biomedicina & \multicolumn{2}{c}{ Agrad. financiamento } & Total \\
\cline { 2 - 3 }
\end{tabular}




\begin{tabular}{|c|c|c|c|}
\hline \multirow[b]{2}{*}{ Citação } & \multirow{2}{*}{\multicolumn{2}{|c|}{ Sem FA }} & \\
\hline & & & \\
\hline Artigos citados (\%) & $58.403(90,44)$ & $13.155(85,95)$ & $71.558(89,58)$ \\
\hline Artigos não citados (\%) & $6.173(9,56)$ & $2.150(14,05)$ & $8.323(10,42)$ \\
\hline Total & 64.576 & 15.305 & 79.881 \\
\hline Ciências Sociais & \multicolumn{2}{|c|}{ Agrad. financiamento } & \multirow[b]{2}{*}{ Total } \\
\hline Citação & Com FA & Sem FA & \\
\hline Artigos citados (\%) & $5.943(80,90)$ & $8.333(77,73)$ & $14.276(79,02)$ \\
\hline Artigos não citados (\%) & $1.403(19,10)$ & $2.387(22,27)$ & $3.790(20,98)$ \\
\hline Total & 7.346 & 10.720 & 18.066 \\
\hline Engenharia, Tecnologia & \multicolumn{2}{|c|}{ Agrad. financiamento } & \multirow[b]{2}{*}{ Total } \\
\hline Citação & Com FA & Sem FA & \\
\hline Artigos citados (\%) & $39.306(86,27)$ & $12.246(79,42)$ & $51.552(84,54)$ \\
\hline Artigos não citados (\%) & $6.256(13,73)$ & $3.173(20,58)$ & $9.429(15,46)$ \\
\hline Total & 45.562 & 15.419 & 60.981 \\
\hline Física & \multicolumn{2}{|c|}{ Agrad. financiamento } & \multirow[b]{2}{*}{ Total } \\
\hline Citação & Com FA & Sem FA & \\
\hline Artigos citados (\%) & $33.899(88,87)$ & $5.164(79,43)$ & $39.063(87,49)$ \\
\hline Artigos não citados (\%) & $4.247(11,13)$ & $1.337(20,57)$ & $5.584(12,51)$ \\
\hline Total & 38.146 & 6.501 & 44.647 \\
\hline Humanidades & \multicolumn{2}{|c|}{ Agrad. financiamento } & \multirow[b]{2}{*}{ Total } \\
\hline Citação & Com FA & Sem FA & \\
\hline Artigos citados (\%) & $159(66,25)$ & $485(45,93)$ & $644(49,69)$ \\
\hline Artigos não citados (\%) & $81(33,75)$ & $571(54,07)$ & $652(50,31)$ \\
\hline Total & 240 & 1.056 & 1.296 \\
\hline Matemática & \multicolumn{2}{|c|}{ Agrad. financiamento } & \multirow[b]{2}{*}{ Total } \\
\hline Citação & Com FA & Sem FA & \\
\hline Artigos citados (\%) & $7.544(74,70)$ & $2.116(71,29)$ & $9.660(73,93)$ \\
\hline Artigos não citados (\%) & $2.555(25,30)$ & $852(28,71)$ & $3.407(26,07)$ \\
\hline Total & 10.099 & 2.968 & 13.067 \\
\hline Medicina Clínica & \multicolumn{2}{|c|}{ Agrad. financiamento } & \\
\hline Citação & Com FA & Sem FA & Total \\
\hline Artigos citados (\%) & $42.326(90,54)$ & $32.316(83,77)$ & $74.642(87,48)$ \\
\hline Artigos não citados (\%) & $4.424(9,46)$ & $6.260(16,23)$ & $10.684(12,52)$ \\
\hline Total & 46.750 & 38.576 & 85.326 \\
\hline Multidisciplinar & \multicolumn{2}{|c|}{ Agrad. financiamento } & \\
\hline Citação & Com FA & Sem FA & Total \\
\hline Artigos citados $(\%)$ & $5.961(86,96)$ & $830(73,06)$ & $6.791(84,98)$ \\
\hline Artigos não citados (\%) & $894(13,04)$ & $306(26,94)$ & $1.200(15,02)$ \\
\hline Total & 6.855 & 1.136 & 7.991 \\
\hline Química & \multicolumn{2}{|c|}{ Agrad. financiamento } & \\
\hline Citação & Com FA & Sem FA & Total \\
\hline Artigos citados (\%) & $23.393(91,08)$ & $3.933(86,71)$ & $27.326(90,42)$ \\
\hline Artigos não citados (\%) & $2.292(8,92)$ & $603(13,29)$ & $2.895(9,58)$ \\
\hline
\end{tabular}




\begin{tabular}{cccc}
\hline Agric., Biol. e M. Amb. & \multicolumn{2}{c}{ Agrad. financiamento } & \multirow{2}{*}{ Total } \\
\cline { 2 - 3 } Citação & Com FA & Sem FA & $72.068(81,10)$ \\
\hline Artigos citados $(\%)$ & $56.859(83,50)$ & $15.209(73,24)$ & $16.799(18,90)$ \\
Artigos não citados $(\%)$ & $11.243(16,50)$ & $5.556(26,76)$ & 30.221 \\
\hline Total & 25.685 & 4.536 & \\
\hline
\end{tabular}

Fonte: Web of Science (2017).

Nota: Os percentuais entre parênteses referem-se aos totais da variável agradecimento por financiamento (totais das colunas). Baseado em uma tabela de contingência $2 \times 2$, o teste $\mathrm{x}^{2}$ quiquadrado de Pearson incluiu artigos originais e artigos de revisão.

$O$ fato de que trabalhos que indicam serem oriundos de pesquisa financiada (artigos Com FA) apresentar um impacto maior do que aqueles de pesquisa não financiada (artigos Sem $F A$ ) já foi evidenciado por diversos especialistas ao analisarem as publicações mundiais de 2009 indexadas pela WoS (COSTAS; VAN LEEUWEN, 2012) e as publicações de 1998 nos sete periódicos mais importantes da área da Biblioteconomia e Ciência da Informação (ZHAO, 2010). Publicações financiadas por agências de fomento reconhecidas geram um maior interesse de citação (WANG; SHAPIRA, 2011). Por outro lado, cientistas como Cronin e Shaw (1999), ao analisarem os artigos de quatro periódicos da Ciência da Informação (1989-1993), comprovaram estatisticamente que as citações estão associadas ao periódico de publicação e à nacionalidade de um autor, mas não ao financiamento.

\section{Considerações finais}

Por meio de indicadores bibliométricos, foi possível analisar a presença de agradecimentos por financiamento - funding acknowledgements (FA) - nos artigos brasileiros de 2009-2016 indexados pela WoS, observando características da pesquisa financiada por área (e disciplinas) no contexto de investigação nacional. Tendo em vista que o apoio recebido por fontes de financiamento é reconhecido em $71,4 \%$ das publicações que compõem o corpus da investigação, considera-se que a análise de FA por área pode gerar resultados confiáveis e relevantes. A presença de agradecimentos por financiamento em artigos científicos por área pode estar condicionada, dentre diversos fatores, à obrigatoriedade (ou não) das agências de fomento, no que se refere à inclusão de 
uma seção no documento reconhecendo o apoio recebido durante a pesquisa que finalizou em publicação. Por outro lado, devido a que o reconhecimento é uma atividade voluntária, os autores das publicações podem decidir não agradecer os diferentes tipos de apoio recebidos, ou esquecer de fazê-lo (COSTAS; VAN LEEUWEN, 2012), deduzindo-se que, provavelmente, alguns dos artigos sem FA possam ter sido de fato financiados. Apesar dessas limitações, entende-se que o presente estudo pode fornecer um amplo panorama sobre a presença de agradecimentos por financiamento como indicadores de pesquisa financiada no Brasil, com especial foco nas diferenças de FA entre áreas.

A maior presença de FA observada em periódicos Q1, quando comparada à da totalidade de periódicos (todos os quartis) e em artigos minimamente citados, quando comparada à da totalidade de artigos sem FA minimamente citados, pode ser considerada um indicador de qualidade da pesquisa financiada no Brasil. De modo geral, a presença de FA em disciplinas de natureza teórica e social de áreas como Ciências Sociais e Humanidades é significativamente baixa em comparação às disciplinas de áreas de natureza experimental e aplicada como Física e Química e indica uma menor dependência de financiamento das primeiras, em concordância com Díaz-Faes e Bordons (2014). Os resultados revelam uma maior presença de FA em artigos de múltipla autoria, provavelmente como consequência da complexidade e custo financeiro das investigações no interior dos teamworks. Tal indicador pode justificar parcialmente a prevalência de publicações financiadas em periódicos Q1 e de publicações financiadas minimamente citadas, uma vez que foi comprovada na literatura uma correlação positiva forte entre o número de autores/artigo e o impacto (LOW et al., 2014). Teoricamente, os indicadores bibliométricos do estudo apontaram que a frequência de agradecimentos por financiamento nos artigos brasileiros varia conforme a área, provavelmente, em virtude das particularidades do conjunto de disciplinas/campos (natureza e orientação da pesquisa, perfil de publicação, estrutura organizacional etc.) que constituem cada uma destas.

Dado que os agradecimentos presentes nos artigos representam um fenômeno social, a continuidade das investigações pode contribuir 
substancialmente para o entendimento das características da colaboração científica com auxílio de indicadores bibliométricos "não tradicionais", analisando padrões de "subautoria" através da análise dos tipos de apoio agradecido pelos autores de natureza "não financeira" (moral, participantes da pesquisa, editorial, técnico/instrumental, Conceitual/PIC - Peer Interactive Communication). Complementarmente, análises qualitativas, baseadas em depoimentos, podem ser relevantes para descobrir motivações (profissionais, acadêmicas, sociais) e razões individuais dos autores das publicações para agradecerem a contribuição material e/ou intelectual recebida durante o desenvolvimento da pesquisa científica e para explorar o comportamento e a percepção da prática por área do conhecimento.

\section{Referências}

ALVAREZ, Gonzalo Rubén; CAREGNATO, Sônia Elisa. Presença de agradecimentos por financiamento nos artigos brasileiros indexados na Web of Science (2009-2016). In: ENCONTRO BRASILEIRO DE BIBLIOMETRIA E CIENTOMETRIA, 6., 2018, Rio de Janeiro. Anais... Rio de Janeiro: UFRJ, 2018.

ALVAREZ, Gonzalo Rubén; VANZ, Samile Andréa de Souza; BARBOSA, Márcia Cristina Bernardes. Scientometric indicators for Brazilian research on High Energy Physics, 1983-2013. Anais da Academia Brasileira de Ciências, Rio de Janeiro, v. 89, n. 3, supl. p. 2525-2543, 2017.

ÁLVAREZ-BORNSTEIN, Belén; MORILLO, Fernanda; BORDONS, María. Funding acknowledgments in the Web of Science: completeness and accuracy of collected data. Scientometrics, Amsterdam, v. 112, n. 3, p. 1793-1812, 2017.

BORDONS, María. et al. La actividad científica del CSIC a través de indicadores bibliométricos (Web of Science, 2011-2015). Madrid: CSIC, 2016.

BOURDIEU, Pierre. Os usos sociais da ciência: por uma sociologia clínica do campo científico. São Paulo: Editora UNESP, 2004.

BOZEMAN, Barry; LEE, Sooho. The impact of research collaboration on scientific productivity. In: ANNUAL MEETING OF THE AMERICAN ASSOCIATION FOR THE ADV ANCEMENT OF SCIENCE, 2003, Denver. Proceedings... Denver: AAAS, 2003. 
BRASIL. Ministério de Ciência e Tecnologia. Recursos Aplicados - Governo Federal. Brasília, DF: MCTI, 2013. Disponível em:

http://www.mctic.gov.br/mctic/opencms/indicadores/detalhe/recursos_aplicados /indicadores_consolidados/2_1_6.html. Acesso em: 7 fev. 2018.

CHOMPALOV, Ivan; GENUTH, Joel; SHRUM, Wesley. The organization of scientific collaborations. Research Policy, v 31, p. 749-767, 2002.

CHUBIN, Daryl E. Trusted assessorship in science: a relation in need of data. Social Studies of Science, v. 5, n. 3, p. 362-367, 1975.

CNPq - CONSELHO NACIONAL DE DESENVOLVIMENTO CIENTÍFICO E TECNOLÓGICO. Investimentos do CNPq em CT\&I. 2018. Disponível em: http://fomentonacional.cnpq.br/dmfomento/home/fmtvisualizador.jsp. Acesso em: 13 mar. 2018.

COSTAS, Rodrigo; LEEUWEN, Thed N. van. Approaching the "reward triangle": General analysis of the presence of funding acknowledgments and "peer interactive communication" in scientific publications. Journal of the American Society for Information Science and Technology, v. 63, n. 8, p. 1647-1661, 2012.

CRONIN, Blaise; SHAW, Debora. Citation, funding acknowledgement and author nationality relationships in four information science journals. Journal of Documentation, v. 55, n. 4, p. 402-408, 1999.

CRONIN, Blaise; SHAW, Debora; LA BARRE, Kathryn. Visible, less visible, and invisible work: patterns of collaboration in 20th century Chemistry. Journal of the American Society for Information Science and Technology, v. 55, n. 2, p. 160-168, 2004.

DÍAZ-FAES, Adrián Arias; GALINDO, María Purificación; BORDONS, María. Nuevas aproximaciones a la colaboración científica: el análisis de los agradecimentos. In: ALCAIDE, Gregorio González; FERRI, Javier Gómez; CALATAYUD, Víctor Agulló (Coord.). La colaboración científica: una aproximación multidisciplinar. Valencia: Nau Llibres, 2013.

DÍAZ-FAES, Adrián Arias; BORDONS, María. Acknowledgments in scientific publications: presence in Spanish science and text patterns across disciplines. Journal of the Association for Information Science and Technology, v. 65, n. 9, p. 1834-1849, 2014.

GILLETT, Raphael. Pitfalls in assessing research performance by grant income. Scientometrics, Amsterdam, v. 22, n. 2, p. 253-263, 1991.

GONZÁLEZ-ALBO, Borja. et al. Bibliometric indicators for the analysis of the research performance of a multidisciplinary institution: the CSIC. Revista Española de Documentación Científica, v. 35, n. 1, p. 9-37, 2012. 
HEFFNER, Alan. Funded research, multiple authorship, and subauthorship collaboration in four disciplines. Scientometrics, Amsterdam, v. 3, n. 1, p. 5-12, 1981.

KATZ, J. Sylvan; MARTIN, Ben R. What is research collaboration? Research Policy, Amsterdam, n. 26, p. 1-18, 1997.

LATOUR, Bruno; WOOLGAR, Steve. A vida de laboratório: a produção dos fatos científicos. Rio de Janeiro: Relume Dumará, 1997.

LAUDEL, Grit. Is external research funding a valid indicator for research performance? Research Evaluation, v. 14, n. 1, p. 27-34, 2005.

LIU, Weishu et al. China's global growth in social science research: uncovering evidence from bibliometric analyses of SSCI publications (1978-2013). Journal of Informetrics, v. 9, n. 3, p. 555-569, 2015.

LETA, Jacqueline; CHAIMOVICH, Hernan. Recognition and international collaboration: the Brazilian case. Scientometrics, Amsterdam, v. 53, n. 3, p. 325-335, 2002.

LEWISON, Grant; GRANT, Jonathan; JANSEN, Paul. International gastroenterology research: subject areas, impact, and funding. Gut, v. 49, n. 2, p. 295-302, 2001.

LEWISON, Grant; MARKUSOVA, Valentina. The evaluation of Russian cancer research. Research Evaluation, v. 19, n. 2, p. 129-144, 2010.

LEWISON, Grant; ROE, Philip. The evaluation of Indian cancer research, 19902010. Scientometrics, Amsterdam, v. 93, n. 1, p. 167-181, 2012.

LOW, Wah Yun et al. Trend and impact of international collaboration in clinical medicine papers published in Malaysia. Scientometrics, Amsterdam, v. 98, n. 2, p. 1521-1533, 2014.

PAUL-HUS, Adèle; DESROCHERS, Nadine; COSTAS, Rodrigo.

Characterization, description, and considerations for the use of funding acknowledgement data in Web of Science. Scientometrics, Amsterdam, v. 108, n. 1, p. 167-182, 2016.

PAUL-HUS, Adèle et al. Acknowledgment research genealogy for today's quantified academia. In: ANNUAL CONFERENCE OF THE CANADIAN ASSOCIATION FOR INFORMATION SCIENCE, 43., 2015, Ottawa.

Proceedings... Ottawa: CAIS, 2015.

RIGBY, John. Systematic grant and funding body acknowledgement data for publications: new dimensions and new controversies for research policy and evaluation. Research Evaluation, v. 20, n. 5, p. 365-375, 2011. 
RIGBY, John. Looking for the impact of peer review: does count of funding acknowledgements really predict research impact? Scientometrics, Amsterdam, v. 94, n. 1, p. 57-73, 2013.

VANZ, Samile Andréa de Souza. As redes de colaboração científica no Brasil: (2004-2006). 2009. 204 f. Tese (Doutorado) - Faculdade de Biblioteconomia e Comunicação, Universidade Federal do Rio Grande do Sul, Porto Alegre, 2009.

VANZ, Samile Andréa de Souza; STUMPF, Ida Regina Chittó. Scientific output indicators and scientific collaboration network mapping in Brazil. Collnet

Journal of Scientometrics and Information Management, 6, p. 1-20, 2012.

VARGAS, Rosely de Andrade. A produção científica brasileira em Ciências Agrárias indexada na Web of Science: características e redes de colaboração (2000-2011). 2014. 131 f. Dissertação (Mestrado) - Faculdade de

Biblioteconomia e Comunicação, Universidade Federal do Rio Grande do Sul, Porto Alegre, 2014.

WANG, Jue; SHAPIRA, Philip. Funding acknowledgement analysis: an enhanced tool to investigate research sponsorship impacts: the case of nanotechnology. Scientometrics, Amsterdam, v. 87, n. 3, p. 563-586, 2011.

WANG, Xianwen et al. Science funding and research output: a study on 10 countries. Scientometrics, Amsterdam, v. 91, p. 591-599, 2012.

ZHAO, Dangzhi. Characteristics and impact of grant-funded research: a case study of the library and information science field. Scientometrics, Amsterdam, v. 84, n. 2, p. 293-306, 2010.

\title{
Funding acknowledgements in Brazilian scientific output represented in the Web of Science
}

\begin{abstract}
Acknowledgments in papers represent a social phenomenon and offer alternative ways for the study of funded research ("financial" acknowledgments) and of 'hidden' co-authorship ("non-financial" acknowledgments) in scientific output. Analyses funding acknowledgments (FA) identified in papers by Brazilian authors published from 2009 to 2016 and indexed in the Web of Science (WoS), observing characteristics of the research by discipline. The corpus is composed of 268,779 papers, of which $71.4 \%$ include information on funding acknowledgments. The greater presence of FA in Q1 journals, when compared to the totality of journals (all quartiles) and minimally cited articles, when compared to the totality of articles without FA, can be considered a quality indicator of the Brazilian funded research. In general, the presence of FA in theoretical and social areas such as Social Sciences and Humanities is
\end{abstract}


significantly low when compared to disciplines in experimental and applied sciences such as Physics and Chemistry and indicates a lower reliance on funding in the former. The results reveal a greater presence of FA in papers of multiple authorship, probably as a result of the complexity and financial cost of teamwork investigations. It concludes that the presence of funding acknowledgments in Brazilian papers varies according to the area due to their particularities, such as nature and orientation of the research, publication profile, organizational structure, among others.

Keywords: Scientific output. Funding acknowledgements. Funded research. Brazilian science. Bibliometrics.

Recebido: 19/09/2018

Aceito: 11/12/2018 